\section{Risk Factors and the Prevention of Weight Gain During Induction Chemotherapy in Children With Acute Lymphoblastic Leukemia}

\section{To the Editor:}

In a recent issue of the Journal of Pediatric Hematology/Oncology, Seki and colleagues reported on risk factors for weight gain during induction chemotherapy in 96 childhood acute lymphoblastic leukemia (ALL) patients. In a retrospective study of medical records, they found that non-high-risk treatment for childhood ALL and treatment start date before daily weight measurement were risk factors for weight gain during induction therapy. They concluded that daily body weight measurements might prevent weight gain during induction therapy and can result in maintaining a healthy weight after ALL treatment. ${ }^{1}$

We agree that early weight management should be emphasized, but question the study's conclusions because of its methodological weaknesses. The retrospective design could have introduced surveillance bias and confounding. During the second half of the study period (after 2005), more focus was put on weight management as daily body weight measurements were initiated in an attempt to control weight. Patients who experienced weight gain in the second half of the study (after 2005) may have been more closely monitored than patients in the first half of the study (before 2005). The closer surveillance could potentially have led to difference in management, for example, diet control and water retention. Reverse causation may have occurred: overall differences in weight management, not the daily weight measurements, caused a weight reduction. More details on how weight gain found during induction was handled in the 2 periods would have helped the reader interpret this study. ${ }^{2}$ In the future, an intervention study with weight monitoring procedures in addition to normal clinical practice of daily weight measurements would assist gaining insight into good management of weight reduction.

The potential misclassification of the outcomes of overweight and obesity is another bias to be considered. Seki and colleagues based their body mass index classification on the International Obesity Taskforce (IOTF) cut-offs defined in 2000. ${ }^{3}$ In 2012, the IOTF published new cut-offs restricted to the age-range 2 to 18 years. ${ }^{4}$ Although these new cut-offs would have only marginally affected the estimated overweight and obesity prevalence, because numbers already were low it would have been more accurate to have used the newest cut-offs together with the body mass index $z$-score cut-offs defined by the World Health Organization for those younger than 2 years. Finally, selective attrition bias might have occurred in the subgroup analyses of 40 patients who did not relapse or have hematopoietic stem cell transplantation because those who were excluded seem to have had a poorer disease status and were more likely be high risk patients. This could have resulted in an underestimate because intense treatment can lead to weight gain suppression.

We think the results of Seki and colleagues need to be interpreted with caution. The power of the study would have been greater had it had a larger study population and the analyses been performed with weight gain as a linear instead of binary variable. Weight management is complex; it should encourage a healthy lifestyle that includes a balanced diet and sufficient physical exercise. Performing only weight measurements may increase awareness but not lead to the long-term lifestyle changes that are essential in maintaining a healthy weight.

Fabiën N. Belle, MSc*†

Rahel Kasteler, MD*

*Institute of Social and Preventive Medicine University of Bern, Switzerland $\dagger$ Institute of Social and Preventive Medicine (IUMSP), Lausanne University Hospital CHUV, Switzerland

\section{REFERENCES}

1. Seki Y, Okamoto Y, Kodama Y, et al. Risk factors and the prevention of weight gain during induction chemotherapy in children with acute lymphoblastic leukemia. J Pediatr Hematol Oncol. 2018;40: e334 e334. [Epub ahead of print].

2. Pannucci CJ, Wilkins EG. Identifying and avoiding bias in research. Plast Reconstr Surg. 2010;126:619-625.

3. Cole TJ, Bellizzi MC, Flegal KM, et al Establishing a standard definition for child overweight and obesity worldwide: international survey. BMJ. 2000;320: $1-6$

4. Cole TJ, Lobstein T. Extended international (IOTF) body mass index cut-offs for thinness, overweight and obesity. Pediatr Obes. 2012;7:284-294. 